\title{
Updates on the risk factors of acute kidney injury after transcatheter aortic valve replacement
}

\author{
Wisit Cheungpasitporn ${ }^{1}$, Charat Thongprayoon ${ }^{2}$, Kianoush Kashani ${ }^{1,3^{*}}$ \\ ${ }^{1}$ Division of Nephrology and Hypertension, Department of Medicine, Mayo Clinic, Rochester, MN, USA \\ ${ }^{2}$ Department of Internal Medicine, Bassett Medical Center, Cooperstown, NY, USA \\ ${ }^{3}$ Division of Pulmonary and Critical Care Medicine, Department of Medicine, Mayo Clinic, Rochester, MN, USA
}

\section{A R T I C L E IN F O}

Article Type:

Authors' Reply

\section{Article History:}

Received: 18 September 2016

Accepted: 19 October 2016

Published online: 24 October 2016

Keywords:

Acute kidney injury

Transcatheter aortic valve replacement Balloon valvuloplasty

\begin{abstract}
Implication for health policy/practice/research/medical education: In our recent updated studies, we found a significant association between periprocedural blood transfusion and acute kidney injury (AKI) following transcatheter aortic valve replacement (TAVR) with an overall 1.95-fold increased the risk of AKI. We also demonstrated that a transapical approach was significantly associated with increased AKI risk compared with a transfemoral approach. Nevertheless, the TAVR approach did not affect severe renal outcomes or long-term renal function. In addition, our meta-analysis demonstrated no significant association between contrast media volume and risk of AKI after TAVR. Thus, the dose of contrast media likely does not play a significant role in the pathogenesis of TAVR-related AKI. Growing knowledge of these risk factors of TAVR on kidney function will help improve preventive measures to improve patients' outcomes. Please cite this paper as: Cheungpasitporn W, Thongprayoon C, Kashani K. Updates on the risk factors of acute kidney injury after transcatheter aortic valve replacement. J Renal Inj Prev. 2017;6(1):16-17. DOI: 10.15171/jrip.2017.03
\end{abstract}

\section{Dear Editor,}

We agree with Onuigbo and Agbasi that previous studies have attempted to identify effective interventions to prevent postoperative acute kidney injury (AKI) events including cardiac surgery-associated AKI and most of them failed to be successful $(1,2)$. In the setting of transcatheter aortic valve replacement (TAVR), we recently performed a comprehensive review of available data on incidence and risk factors of AKI after TAVR (3). We demonstrated that causes of AKI following TAVR are likely multifactorial including hypotension during rapid ventricular pacing for balloon valvuloplasty and valve deployment, use of contrast agents, and embolization resulting from the manipulation of catheters in the aorta of patients with diffuse atherosclerosis (4-6). However, we found that the data on effects of transapical (TA) vs. transfemoral (TF) approaches, contrast media and blood transfusion on AKI following TAVR were still controversial. Thus, we performed additional studies to evaluate these effects on AKI after TAVR, and ultimately to prevent AKI. We recently conducted a meta-analysis of 17 cohort studies with 5,085 patients assessing the risk of AKI in patients undergoing TA-TAVR vs. TF-TAVR (7). We found a significant association between TA-TAVR and a higher risk of AKI. However, the risk of severe AKI in patients who underwent TA-TAVR compared with TF-TAVR was not significantly higher. We subsequently performed a propensity-adjusted analysis of 366 patients undergoing TAVR to compare the incidence of post-procedural AKI following TA-TAVR vs. TF-TAVR (8). We confirmed that a TA approach was significantly associated with increased AKI risk compared with a TF approach. Nevertheless, the TAVR approach did not affect severe renal outcomes or long-term renal function. Regarding the effects of contrast media volume on TAVR-related AKI, we recently analyzed the data from 4 cohort studies comprised of 891 patients undergoing TAVR (9). Interestingly, our metaanalysis demonstrated no significant association between contrast media volume and risk of AKI after TAVR. Although the finding in this study might be limited by the lack of statistical power in detecting clinically meaning the effect of contrast volume on AKI, given a small number of included studies, these effects were small. Thus, the dose of contrast media likely does not play a significant role in the pathogenesis of TAVR-related AKI. Lastly, our recent meta-analysis of 16 cohort studies with 4690 
patients assessing the risk of AKI after TAVR in patients who received a periprocedural blood transfusion (10). In this study, we found a significant association between periprocedural blood transfusion and AKI following TAVR with an overall 1.95-fold increased the risk of AKI. Growing knowledge of these risk factors of TAVR on kidney function will help improve preventive measures to improve patients' outcomes.

\section{Authors' contribution}

All authors have contributed equally to the preparation of the manuscript.

\section{Conflicts of interest}

The authors declare no conflict of interest.

\section{Ethical considerations}

Ethical issues (including plagiarism, data fabrication, double publication) have been completely observed by the authors.

\section{Funding/Support}

None.

\section{References}

1. Cheng X, Tong J, Hu Q, Chen S, Yin Y, Liu Z. Metaanalysis of the effects of preoperative renin-angiotensin system inhibitor therapy on major adverse cardiac events in patients undergoing cardiac surgery. Eur J Cardiothorac Surg. 2015;47:958-66. doi: 10.1093/ejcts/ezu330.

2. Yacoub R, Patel N, Lohr JW, Rajagopalan S, Nader N, Arora P. Acute kidney injury and death associated with renin angiotensin system blockade in cardiothoracic surgery: a meta-analysis of observational studies. Am J Kidney Dis. 2013;62:1077-86.

3. Cheungpasitporn W, Thongprayoon C, Kashani K. Transcatheter aortic valve replacement: a kidney's perspective. J Renal Inj Prev. 2016;5:1-7. doi: 10.15171/ jrip.2016.01

4. Thongprayoon C, Cheungpasitporn W, Srivali N, Ungprasert P, Kittanamongkolchai W, Greason KL, et al. Acute kidney injury after transcatheter aortic valve replacement: a systematic review and meta-analysis. Am J Nephrol. 2015;41:372-82. doi: 10.1159/000431337.

5. Thongprayoon C, Cheungpasitporn W, Srivali N, Harrison AM, Gunderson TM, Kittanamongkolchai W, et al. AKI after transcatheter or surgical aortic valve replacement. J Am Soc Nephrol. 2016;27:1854-60. doi: 10.1681/asn.2015050577

6. Thongprayoon C, Cheungpasitporn W, Srivali N, Kittanamongkolchai W, Greason KL, Kashani KB. Incidence and risk factors of acute kidney injury following transcatheter aortic valve replacement. Nephrology (Carlton). 2015. doi: 10.1111/nep.12704.

7. Thongprayoon C, Cheungpasitporn W, Gillaspie EA, Greason KL, Kashani KB. The risk of acute kidney injury following transapical versus transfemoral transcatheter aortic valve replacement: a systematic review and metaanalysis. Clin Kidney J. 2016;9:560-6. doi: 10.1093/ckj/ sfw055

8. Thongprayoon C, Cheungpasitporn W, Srivali N, Harrison AM, Kittanamongkolchai W, Greason KL, et al. Transapical versus transfemoral approach and risk of acute kidney injury following transcatheter aortic valve replacement: a propensity-adjusted analysis. Ren Fail. 2016. doi: 10.1080/0886022x.2016.1244072.

9. Thongprayoon C, Cheungpasitporn W, Podboy AJ, Gillaspie EA, Greason KL, Kashani KB. The effects of contrast media volume on acute kidney injury after transcatheter aortic valve replacement: a systematic review and meta-analysis. J Evid Based Med. 2016. doi: 10.1111/jebm.12208.

10. Thongprayoon C, Cheungpasitporn W, Gillaspie EA, Greason KL, Kashani KB. Association of blood transfusion with acute kidney injury after transcatheter aortic valve replacement: A meta-analysis. World J Nephrol. 2016;5:4828. doi: $10.5527 /$ wjn.v5.i5.482.

Copyright (c) 2017 The Author(s); Published by Nickan Research Institute. This is an open-access article distributed under the terms of the Creative Commons Attribution License (http://creativecommons.org/licenses/by/4.0), which permits unrestricted use, distribution, and reproduction in any medium, provided the original work is properly cited. 